\title{
Valproic Acid Prevents NMDA-Induced Retinal Ganglion Cell Death via Stimulation of Neuronal TrkB Receptor Signaling
}

\author{
Atsuko Kimura, Kazuhiko Namekata, Xiaoli Guo, Takahiko Noro, Chikako Harada, and Takayuki Harada
}

From the Visual Research Project, Tokyo Metropolitan Institute of Medical Science, Tokyo, Japan

\author{
Accepted for publication \\ November 10, 2014. \\ Address correspondence to \\ Takayuki Harada, M.D., Ph.D., \\ Visual Research Project, Tokyo \\ Metropolitan Institute of Medical \\ Science, 2-1-6 Kamikitazawa, \\ Setagaya-ku, Tokyo 156-8506, \\ Japan. E-mail: harada-tk@ \\ igakuken.or.jp.
}

\begin{abstract}
Valproic acid (VPA) is widely prescribed for treatment of epilepsy, mood disorders, migraines, and neuropathic pain. It exerts its therapeutic benefits through multiple mechanisms, including enhancement of GABAergic activity, activation of prosurvival protein kinases, and inhibition of histone deacetylase. Increasing evidence suggests that VPA possesses neuroprotective properties. We examined neuroprotective effects of VPA in an N-methyl-D-aspartate (NMDA) excitotoxicity model, which mimics some of the pathological features of glaucoma. In vivo retinal imaging using optical coherence tomography revealed that NMDA-induced retinal degeneration was suppressed in the VPA-treated retina, and histological analyses confirmed that VPA reduced retinal ganglion cell death. In vivo electrophysiological analyses demonstrated that visual impairment was prevented in the VPA-treated retina, clearly establishing both histological and functional effects of VPA. Brain-derived neurotrophic factor (BDNF) expression was up-regulated in Müller glial cells, and neuroprotective effects of VPA on retinal ganglion cells were significantly reduced in a conditional knockout mouse strain with deletion of tropomyosin receptor kinase $B$ (TrkB), a receptor for BDNF from retinal ganglion cells. The results show that VPA stimulates BDNF up-regulation in Müller glial cells and provides direct evidence that neuronal TrkB is important in VPA-mediated neuroprotection. Also, VPA suppresses oxidative stress induced by NMDA in the retina. Our findings raise intriguing possibilities that the widely prescribed drug VPA may be useful for treatment of glaucoma. (Am J Pathol 2015, 185: 756-764; http://dx.doi.org/10.1016/j.ajpath.2014.11.005)
\end{abstract}

Valproic acid (VPA), a short-chain fatty acid, is widely prescribed as an antiepileptic drug. The pharmacological action of VPA in the treatment of epilepsy involves multiple mechanisms, including those associated with regulation of GABAergic neurotransmission, which has been studied since the 1970s. ${ }^{1}$ VPA is also used for treatment of mood disorders, migraines, and neuropathic pain, ${ }^{2-4}$ which indicates both tolerance and clinical importance. The diversity of uses may be explained by the fact that VPA affects numerous systems. Multiple intracellular signal transduction pathways are modulated by VPA, as a result of its action on regulation of enzymatic activities including phosphatidylinositol 3-kinase/ Akt-1, mitogen-activated protein kinases and glycogen synthase kinase $3 \beta$, and histone deacetylase. ${ }^{5-7}$ There is increasing evidence that VPA has neuroprotective properties, and in recent studies VPA has shown promising results in several models of acute injury (including stroke, traumatic brain injury, and spinal cord injury). ${ }^{8}$ VPA also exerts neuroprotective effects in neurodegenerative diseases, including Parkinson disease and Alzheimer disease. ${ }^{9-12}$

Glaucoma, a neurodegenerative disease, is one of the leading causes of vision loss. It is estimated that this condition will affect more than 80 million people worldwide by 2020 , with at least 6 to 8 million of them becoming bilaterally blind. ${ }^{13}$ Glaucoma is characterized by progressive degeneration of retinal ganglion cells (RGCs) and their axons, usually in association with elevated intraocular pressure. There are several animal models of glaucoma, including DBA/2J

\footnotetext{
Supported by the Ministry of Education, Culture, Sports, Science and Technology of Japan (26861479 to A.K., 256400043 to K.N., 25430082 to X.G., 25462766 to C.H., and 26640048 to T.H.) and the Funding Program for Next Generation World-Leading Researchers (NEXT Program) (LS133 to T.H.).

Disclosures: None declared.
} 
mice ${ }^{14}$ and inducible models such as cauterization of episcleral veins. ${ }^{15-17}$ Normal tension glaucoma is a type of glaucoma with statistically normal intraocular pressure; the occurrence rate seems to vary according to race. ${ }^{18,19}$ We have previously reported that loss of glutamate transporters (EAAC1 or GLAST) in mice leads to RGC degeneration similar to normal tension glaucoma, ${ }^{20}$ and these animal models have been useful in examining potential therapeutic targets. ${ }^{21-23}$ Acute models of RGC death are also available, including intravitreal injection of toxins such as $N$-methyl-Daspartate (NMDA) $)^{24,25}$ or staurosporine. ${ }^{26}$ NMDA is a synthetic compound that selectively activates NMDA receptors (which are a subtype of glutamate receptors), and it mimics the action of glutamate. Excessive activation of glutamate receptors induces a steep rise in intracellular calcium levels and causes excitotoxic cell death. Neuroprotection is a key aspect for development of glaucoma therapy. Indeed, inhibition of NMDA receptors has been a potential therapeutic target for glaucoma. The NMDA antagonist memantine showed promising neuroprotective effects in preclinical studies, although it was not successful in recent clinical trials. ${ }^{27-29}$

Brain-derived neurotrophic factor (BDNF) is known to regulate neural cell survival, mainly by activating tropomyosin receptor kinase B (TrkB) receptors. Several lines of evidence support a key role for BDNF-TrkB signaling in survival of adult RGCs in acute and chronic models of optic nerve damage. ${ }^{30,31}$ Gene therapy with BDNF was effective in protecting RGCs in experimental glaucoma, ${ }^{32}$ suggesting the BDNF-TrkB signaling pathway as a potential therapeutic target for treatment of glaucoma.

In the present study, we investigated if VPA protects RGCs from excitotoxic damage using an excitotoxicity model induced by intravitreal injection of NMDA. We examined the effects of VPA using histological techniques as well as in vivo spectral-domain optical coherence tomography and multifocal electroretinography. We provide direct evidence that stimulation of neuronal TrkB receptor signaling is one of the underlying molecular mechanisms for VPA-mediated neuroprotection in the retina.

\section{Materials and Methods}

\section{Animals}

Experiments were performed using C57BL/6J mice (CLEA Japan, Tokyo, Japan) or TrkB ${ }^{\text {flox/flox }}: c-k i t-\mathrm{Cre}^{+}\left(\mathrm{TrkB}^{\mathrm{c}-\mathrm{kit}} \mathrm{KO}\right)$ mice and their WT littermates, ${ }^{33}$ in accordance with the Tokyo Metropolitan Institute of Medical Science Guidelines for the Care and Use of Animals. Light intensity inside the cages ranged from 100 to 200 lux and a 12 hours light/12 hours dark cycle was maintained.

\section{Drug Treatment}

Mice were anesthetized by intraperitoneal injection of 87.5 $\mathrm{mg} / \mathrm{kg}$ sodium pentobarbital, and intravitreal injections were performed under a microsurgical microscope (Olympus Corporation, Tokyo, Japan) using a glass microsyringe with a 33-gauge needle (Ito Corporation, Shizuoka City, Japan). Eyes were punctured at the upper temporal limbus and a volume of $2 \mu \mathrm{L}$ of phosphate-buffered saline (PBS), VPA (75 $\mathrm{mmol} / \mathrm{L}$ in PBS) (Sigma-Aldrich, Tokyo, Japan), NMDA (1 mmol/L in PBS) (Sigma-Aldrich), or NMDA + VPA $(1 \mathrm{mmol} / \mathrm{L}$ and $75 \mathrm{mmol} / \mathrm{L}$ in PBS, respectively) was injected. To allow diffusion of the solution, the needle was kept inside the eye for approximately 1 minute after the delivery. Mice were allowed to fully recover on a heating pad set at $37^{\circ} \mathrm{C}$. The concentrations of the drugs were selected based on our preliminary studies evaluating a range of concentrations (data not shown).

\section{Imaging Acquisition with SD-OCT}

Mice were anesthetized by intraperitoneal injection of 87.5 $\mathrm{mg} / \mathrm{kg}$ sodium pentobarbital, and spectral-domain optical coherence tomography (SD-OCT) examinations using an RS-3000 system (Nidek, Gamagori, Japan) were performed to monitor retinal degeneration in vivo. ${ }^{22,23,34}$ For fundus imaging, polymethyl methacrylate contact lenses optimal for mice (Unicon, Osaka, Japan) were placed on the corneas. Use of the contact lenses prevents anesthesia-induced cataract progression. A 60D adaptor lens was placed on the objective lens of the Multiline OCT to focus on the mouse retina (Volk Opitcal, Mentor, OH). All of the images were location-matched, scanning vertically through the center of the optic nerve head at 3 disk diameter lengths above the optic nerve head. ${ }^{22,34}$ The average thickness of the ganglion cell complex (ie, between the internal limiting membrane and the interface of the inner plexiform layer and the inner nuclear layer) was measured. The maximum number of B-scans set by the manufacturer (ie, 50 for line scans) was used for averaging.

\section{Histological Analysis}

At the end of the experimental period, mice were sacrificed and perfused with Zamboni's fixative (2\% paraformaldehyde and $15 \%$ picric acid in $0.1 \mathrm{~mol} / \mathrm{L}$ phosphate buffer). Eyes were enucleated and postfixed for 2 hours in $3 \%$ glutaraldehyde solution (3\% glutaraldehyde, $9 \%$ formaldehyde, $37.5 \%$ ethanol, and $12.5 \%$ acetic acid in distilled water). Paraffin-embedded retinal sections $(7 \mu \mathrm{m}$ thick) were cut through the optic nerve and stained with hematoxylin and eosin. The extent of retinal degeneration was quantified in two ways. ${ }^{35}$ First, the number of neurons in the ganglion cell layer (GCL) was counted from one ora serrata through the optic nerve to the other ora serrata in a section. Second, in the same section, the thickness of the inner retinal layer (ie, between the internal limiting membrane and the interface of the outer plexiform layer with the outer nuclear layer) was measured. 


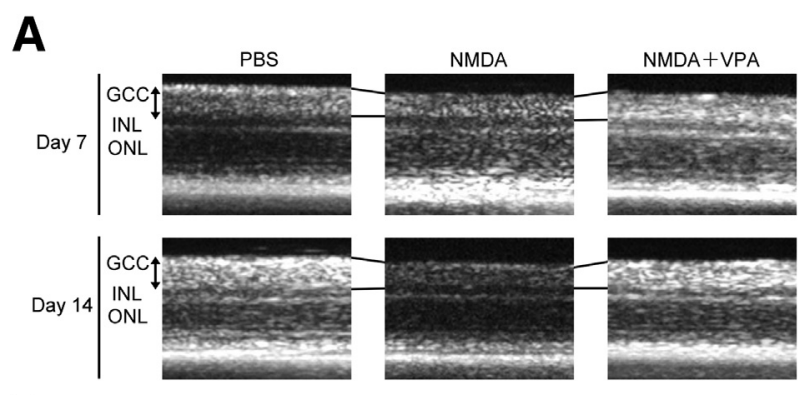

B

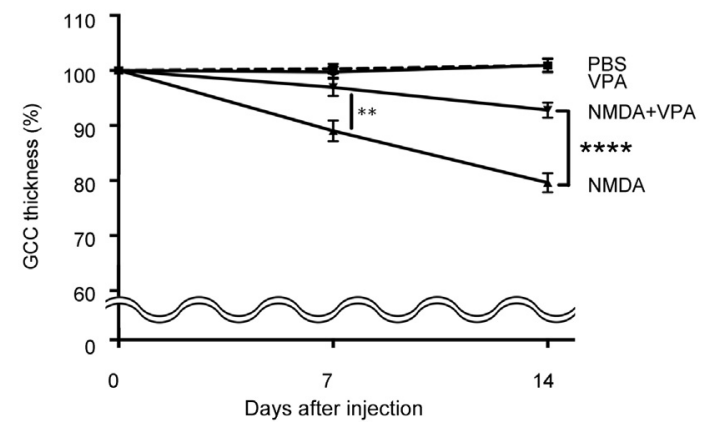

Figure 1 In vivo imaging of the retina demonstrates VPA-mediated suppression of retinal degeneration after NMDA-induced neurotoxicity in mice. A: Representative OCT cross-sectional images of retinas at 7 and 14 days after intravitreal injection of PBS, $75 \mathrm{mmol} / \mathrm{L} \mathrm{VPA}, 1 \mathrm{mmol} / \mathrm{L} \mathrm{NMDA}$, or $1 \mathrm{mmol} / \mathrm{L} \mathrm{NMDA}+75 \mathrm{mmol} / \mathrm{L}$ VPA. B: Corresponding longitudinal evaluation of GCC thickness as a percentage of the PBS control. Data are expressed as means \pm SEM. $n=6 .{ }^{* *} P<0.01,{ }^{* * * *} P<0.0001$. GCC, ganglion cell complex; INL, inner nuclear layer; NMDA, N-methyl-Daspartate; $0 \mathrm{CT}$, optical coherence tomography; $\mathrm{ONL}$, outer nuclear layer; PBS, phosphate-buffered saline; VPA, valproic acid.

\section{Retrograde RGC Labeling}

Mice were deeply anesthetized with isoflurane (Intervet, Tokyo, Japan) and then received intravitreal injection of PBS, NMDA, or NMDA+VPA as described in Drug Treatment. Immediately afterwards, the mice were placed on a stereotaxic frame and received an injection of $2 \mu \mathrm{L}$ FluoroGold (Fluorochrome, Englewood, CO) hydroxystilbamidine ( $2 \%$ in $10 \%$ dimethyl sulfoxide) into the superior colliculus. ${ }^{35}$ At 7 days after Fluoro-Gold application, the mice were sacrificed, eyes were enucleated, and retinas were isolated for whole-mount preparation. Retinas were fixed in $4 \%$ paraformaldehyde in a $0.1 \mathrm{~mol} / \mathrm{L}$ phosphate buffer solution for 1 hour and mounted on a glass slide with a Vectashield mounting medium (Vector Laboratories, Burlingame, CA); the RGC density was then evaluated under a fluorescence microscope. Three standard areas $\left(0.04 \mathrm{~mm}^{2}\right)$ of each retina at a point $0.1 \mathrm{~mm}$ from the optic disk were randomly chosen. Fluoro-Gold-labeled cells were counted, and the average number of RGCs per square millimeter was calculated.

\section{Multifocal Electroretinography}

Mice were anesthetized by intraperitoneal injection of 87.5 $\mathrm{mg} / \mathrm{kg}$ sodium pentobarbital. The pupils were dilated with $0.5 \%$ phenylephrine hydrochloride and $0.5 \%$ tropicamide.
Multifocal electroretinograms were recorded using a VERIS 6.0 system (Electro-Diagnostic Imaging, Redwood City, CA). The visual stimulus consisted of seven hexagonal areas scaled with eccentricity. The stimulus array was displayed on a highresolution black-and-white monitor driven at a frame rate of $100 \mathrm{~Hz}$. The second-order kernel, which is impaired in patients with glaucoma, was analyzed as previously reported. ${ }^{20}$

\section{Cell Culture}

Müller cells were isolated as described previously. ${ }^{36}$ Müller cells derived from WT mice were stimulated with $0,0.1$, or $1 \mathrm{mmol} / \mathrm{L}$ VPA for 12 or 24 hours and processed for quantitative real-time PCR analyses.

\section{Quantitative Real-Time PCR}

Quantitative real-time PCR was performed using an ABI 7300 real-time PCR system (Life Technologies, Carlsbad, CA) with a Power SYBR Green PCR master mix (Life Technologies) as described previously. ${ }^{33,37}$ cDNA reverse-transcribed from total RNA was amplified using primers specific for BDNF (sense, $5^{\prime}$-ATGCCGCAAACATGTCTATGAG-3'; antisense, 5'-TGACCCACTCGCTAATACTGTCA-3'), nerve growth factor (NGF; sense, 5'-CGACTCCAAACACTGGAACTCA-3'; antisense, $5^{\prime}$-GCCTGCTTCTCATCTGTTGTCA-3'), glial cell-derived neurotrophic factor (GDNF; sense, 5'-GGCCTACCTTGTCACTTGTTAGC-3'; antisense, 5'-GGCCTACTTTGTCACTTGTTAGC-3'), ciliary neurotrophic factor (CNTF; sense, 5'-GGTGACTTCCATCAGGCAATACA- $3^{\prime}$; antisense, $5^{\prime}$-CTGTTCCAGAAGCGCCATTAAC- ${ }^{\prime}$ ), inducible nitric oxide synthase (sense, $5^{\prime}$-ACTGTGTGCCTG-

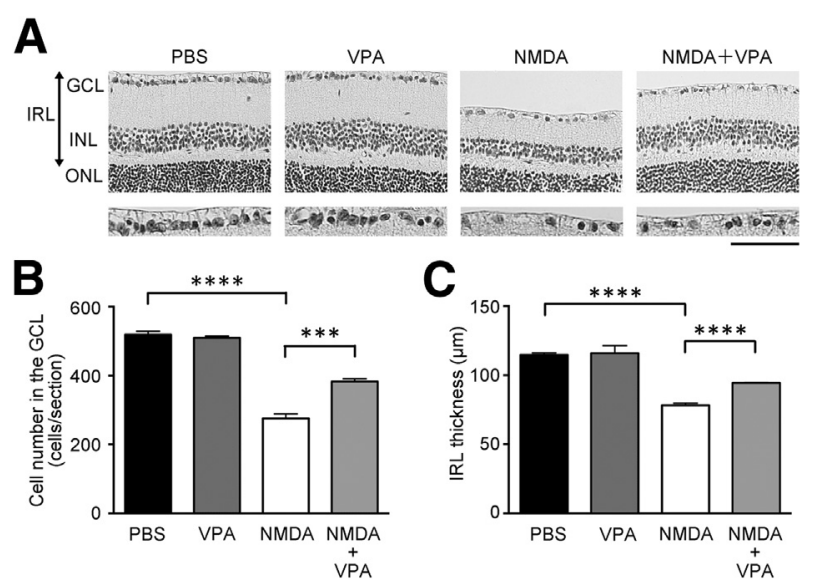

Figure 2 VPA suppresses cell loss in the GCL after NMDA-induced neurotoxicity in mice. A: Representative retinal sections at 14 days after intravitreal injection of PBS, $75 \mathrm{mmol} / \mathrm{L}$ VPA, $1 \mathrm{mmol} / \mathrm{L} \mathrm{NMDA}$, or $1 \mathrm{mmol} / \mathrm{L}$ NMDA $+75 \mathrm{mmol} / \mathrm{L}$ VPA. Hematoxylin and eosin stain. B and C: Corresponding quantitative analyses of cell number per section in the $\mathrm{GCL}(\mathbf{B})$ and IRL thickness (C). Data are expressed as means \pm SEM. $n=6$. ${ }^{* * *} P<0.001,{ }^{* * * * P}<0.0001$. Scale bar $=100 \mu \mathrm{m}$ (upper images, $\mathbf{A}$ ) or $50 \mu \mathrm{m}$ (lower images, A). GCL, ganglion cell layer; INL, inner nuclear layer; IRL, inner retinal layer; NMDA, N-methyl-D-aspartate; $\mathrm{ONL}$, outer nuclear layer; PBS, phosphate-buffered saline; VPA, valproic acid. 

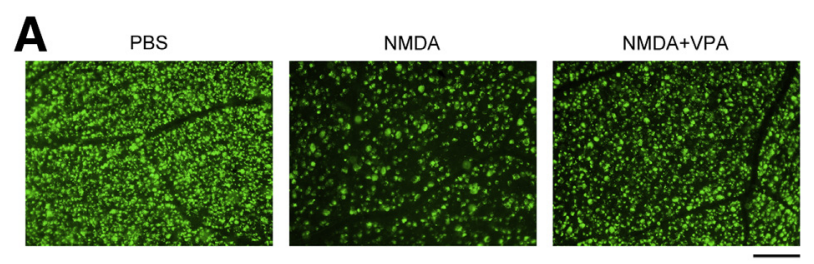

\section{B}

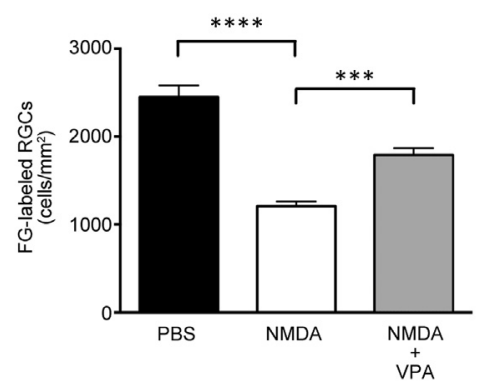

Figure 3 VPA suppresses RGC loss after NMDA-induced neurotoxicity in mice. A: Representative images of retrograde-labeled RGCs at 7 days after intravitreal injection of PBS, $1 \mathrm{mmol} / \mathrm{L} \mathrm{NMDA}$, or $1 \mathrm{mmol} / \mathrm{L} \mathrm{NMDA}+75$ $\mathrm{mmol} / \mathrm{L}$ VPA. B: Corresponding quantitative analyses. Data are expressed as means \pm SEM. $n=3 .{ }^{* * * P}<0.001,{ }^{* * * * P}<0.0001$. Scale bar $=100$ $\mu \mathrm{m}$. FG, Fluoro-Gold hydroxystilbamidine; NMDA, N-methyl-D-aspartate; PBS, phosphate-buffered saline; RGC, retinal ganglion cell; VPA, valproic acid.

GAGGTTCT-3'; antisense, 5'-GGCAGCCTCTTGTCTTTGAC- $3^{\prime}$ ), and GAPDH (sense, $5^{\prime}$-TGCACCACCAACTGCTTAG-3'; antisense, 5'-GGATGCAGGGATGATGTTC-3'). Data were normalized to the level of GAPDH mRNA.

\section{Immunohistochemistry}

Mice received intravitreal injection of PBS, NMDA, or NMDA+VPA as described in Drug Treatment and were perfused with Zamboni's fixative at 12 hours. Eyes were enucleated, postfixed in Zamboni's fixative for 1 hour, and saturated in a sucrose buffer $(30 \%$ sucrose in a $0.1 \mathrm{~mol} / \mathrm{L}$ phosphate buffer). Retinal cryostat sections, $10 \mu \mathrm{m}$ thick, were prepared and examined by immunostaining as described previously, ${ }^{35}$ using a 4-hydroxy-2-nonenal mouse monoclonal antibody (MHN-020P, $2 \mu \mathrm{g} / \mathrm{mL}$ ) (Japan Institute for the Control of Aging, Shizuoka City, Japan).

\section{Statistical Analysis}

For statistical comparison of two samples, we used a twotailed Student's $t$-test. Data are expressed as means \pm SEM. $P<0.05$ was regarded as statistically significant.

\section{Results}

\section{VPA Suppresses NMDA-Induced Retinal Degeneration}

A single intravitreal injection of NMDA induces retinal degeneration, particularly in the GCL, and this experimental strategy is useful for investigating neuroprotective mechanisms that may be effective in glaucoma therapy. ${ }^{24,25}$ To examine the effects of VPA on this NMDA excitotoxicity model, we injected NMDA intravitreally, with or without VPA. First, we used SD-OCT to monitor the degree of retinal degeneration over a course of 2 weeks. SD-OCT acquires cross-sectional tomographic images of the retina noninvasively, allowing monitoring of the retinal morphology in live subjects, and recent advances in technology have enabled its use in experimental animals. The SD-OCT images revealed that the thickness of the ganglion cell complex was significantly reduced at days 7 and 14 after NMDA injection, and these reductions were suppressed in the presence of VPA (Figure 1). We then examined histopathology of the retina after injection of NMDA with or without VPA. A single intravitreal injection of NMDA clearly decreased the number of surviving neurons in the GCL, and this effect was ameliorated by simultaneous injection of VPA (Figure 2, A and $\mathrm{B}$ ). In addition, the thickness of the inner retinal layer was significantly reduced after NMDA injection, and this effect also was suppressed by VPA (Figure 2, A and C). We next specifically labeled RGCs by retrograde labeling with Fluoro-Gold to determine the effects of VPA on RGC survival after NMDA injection. Consistent with the trends observed in the number of cells in the GCL, NMDA induced a significant loss of RGCs and again VPA protected RGCs from this damage (Figure 3). Taken together, these results demonstrate histologically that VPA suppresses retinal degeneration and protects RGCs from NMDA-induced neurotoxicity.
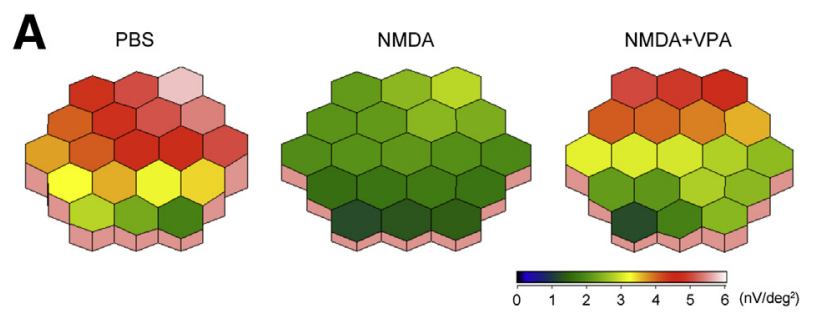

B

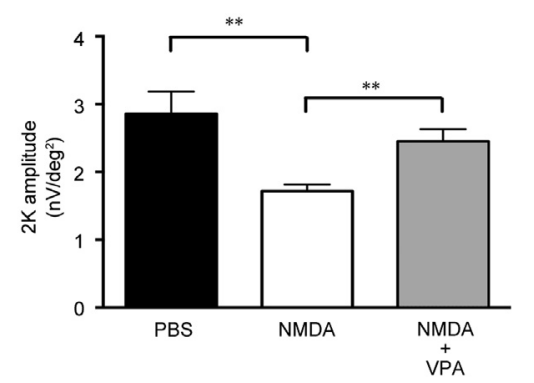

Figure 4 VPA ameliorates NMDA-induced loss of visual function in mice. A: Representative three-dimensional plots of averaged visual responses of the second-order kernel as examined by multifocal electroretinography at 14 days after intravitreal injection of PBS, $1 \mathrm{mmol} / \mathrm{L} \mathrm{NMDA}$, or $1 \mathrm{mmol} / \mathrm{L} \mathrm{NMDA}+75 \mathrm{mmol} / \mathrm{L}$ VPA. Retinal function is scored in nanovolts per degree squared; higher values indicate highly sensitive visual function and lower values indicate retinal dysfunction. B: Corresponding quantitative analyses. Data are expressed as means \pm SEM. $n=6$. ${ }^{*} P<0.01 .2 \mathrm{~K}$, second-order kernel; deg $^{2}$, degree squared; NMDA, N-methyl-D-aspartate; VPA, valproic acid. 

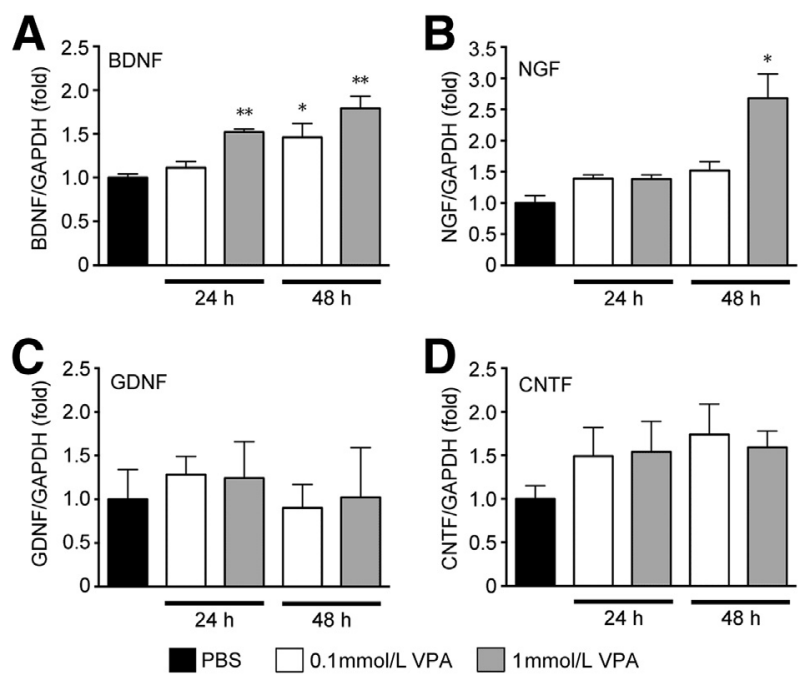

Figure 5 VPA stimulates up-regulation of neurotrophic factors in Müller cells in mice. A-D: Expression of neurotrophic factors in Müller cells at 24 and 48 hours after stimulation with 0.1 or $1 \mathrm{mmol} / \mathrm{L}$ VPA. Relative mRNA expression levels of BDNF (A), NGF (B), GDNF (C), and CNTF (D). GAPDH was used as an internal control and results are normalized to controls (GAPDH mRNA expression). Data are expressed as means \pm SEM. $n=4 .{ }^{*} P<0.05$, ${ }^{*} P<0.01$. BDNF, brain-derived neurotrophic factor; CNTF, ciliary neurotrophic factor; GDNF, glial cell-derived neurotrophic factor; NGF, nerve growth factor; PBS, phosphate-buffered saline; VPA, valproic acid.

\section{VPA Ameliorates NMDA-Induced Loss of Visual} Function

To determine if the observed changes in cell numbers due to NMDA with and without VPA reflect functional aspects, we examined visual function using multifocal electroretinography, which is a vital tool for substantiating histological observations with physiological phenomena in the retina. Multifocal electroretinography is an established noninvasive method for effectively measuring retinal activity in living animals, and it allows isolation of the RGC contribution. ${ }^{20,38}$ We analyzed the second-order kernel, which appears to be a sensitive indicator of inner retinal dysfunction and is impaired in glaucoma patients. ${ }^{39}$ The response topography demonstrating the second-order kernel component revealed that NMDA significantly impaired visual responses in all visual fields at day 14, compared with vehicle, and VPA markedly suppressed these effects (Figure 4). These results point to a very important conclusion, that the neuroprotective effects of VPA after NMDA-induced neurotoxicity are functionally significant.

\section{VPA Promotes Up-Regulation of Neurotrophic Factors in Müller Glial Cells}

We have previously identified important roles of Müller glial neurotrophic factors in protection of retinal neurons. ${ }^{23,33,36}$ We therefore speculated that one of the VPA-mediated neuroprotective mechanisms might involve up-regulation of neurotrophic factors in Müller cells and, in particular, that an increase in neurotrophic factor levels in Müller cells might in turn stimulate survival of RGCs. VPA treatment produced a time- and dose-dependent increase in BDNF expression in cultured Müller cells, and the higher dose of VPA $(1 \mathrm{mmol} / \mathrm{L})$ also up-regulated NGF expression at 48 hours (Figure 5, A and B). VPA at the doses and time points investigated did not affect the expression level of GDNF or CNTF (Figure 5, C and D). These results suggest that VPA up-regulates BDNF and NGF in Müller cells and exerts neuroprotective effects on RGCs indirectly.

\section{VPA Protects RGCs through Neuronal TrkB Signaling}

The VPA-stimulated up-regulation of neurotrophic factors in Müller cells suggested that VPA may indirectly activate the corresponding signaling pathways in RGCs for protection against NMDA-induced neurotoxicity. Because BDNF showed a significant dose-dependent up-regulation, we focused on the BDNF signaling pathway. To examine the role of neuronal BDNF-TrkB signaling in VPAmediated neuroprotection, we used conditional knockout (KO) mice in which TrkB is deleted from two types of retinal neurons, RGCs and amacrine cells $\left(\right.$ TrkB $^{\mathrm{c}-\mathrm{kit}} \mathrm{KO}$ mice). ${ }^{33}$ We first monitored the effects of VPA on retinal degeneration after NMDA injection in $\mathrm{TrkB}^{\mathrm{c}-\mathrm{kit}} \mathrm{KO}$ mice
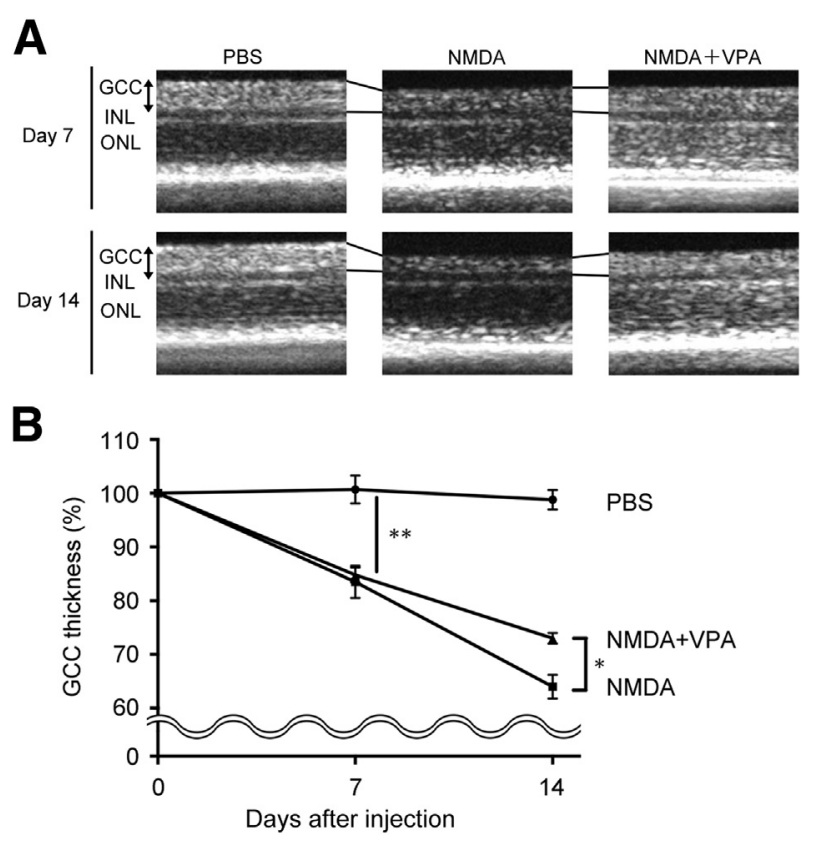

Figure 6 In vivo imaging of the retina demonstrates that VPA-mediated suppression of retinal degeneration is significantly reduced in $\operatorname{TrkB}^{\mathrm{c}-\mathrm{kit}}$ knockout mice. A: Representative OCT cross-sectional images of TrkB ${ }^{\mathrm{c}-\mathrm{kit}}$ knockout mouse retinas at 7 and 14 days after intravitreal injection of PBS, $1 \mathrm{mmol} / \mathrm{L} \mathrm{NMDA}$, or $1 \mathrm{mmol} / \mathrm{L} \mathrm{NMDA}+75 \mathrm{mmol} / \mathrm{L}$ VPA. B: Corresponding longitudinal evaluation of GCC thickness as a percentage of the PBS control. Data are expressed as means \pm SEM. $n=6$. ${ }^{*} P<0.05,{ }^{*} P<0.01$. GCC, ganglion cell complex; INL, inner nuclear layer; NMDA, N-methyl-Daspartate; $\mathrm{OCT}$, optical coherence tomography; $\mathrm{ONL}$, outer nuclear layer; PBS, phosphate-buffered saline; VPA, valproic acid. 

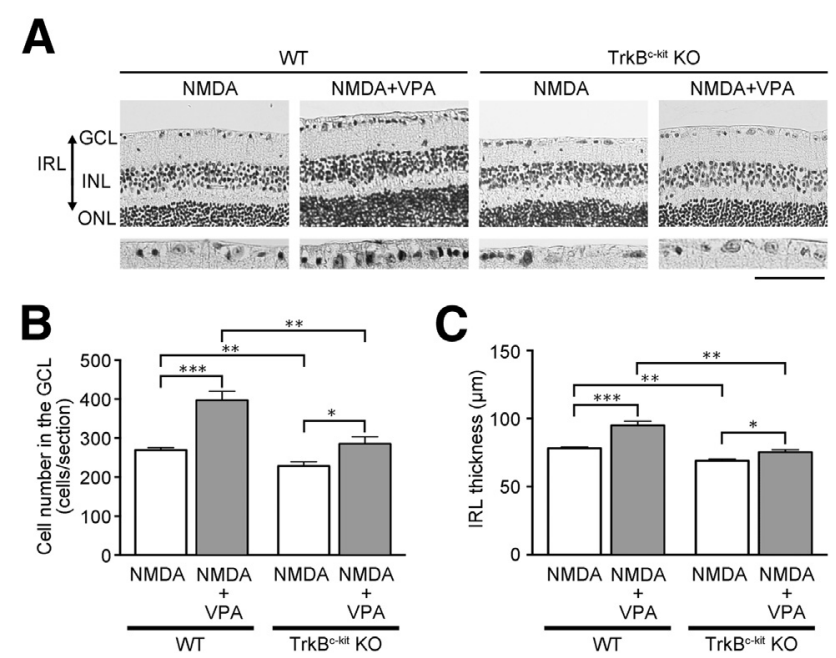

Figure 7 VPA-mediated protection of cells in the GCL is significantly reduced in $\mathrm{TrkB}^{\mathrm{c}-\mathrm{kit}} \mathrm{KO}$ mice. A: Representative retinal sections at 14 days after intravitreal injection of $1 \mathrm{mmol} / \mathrm{L}$ NMDA or $1 \mathrm{mmol} / \mathrm{L}$ NMDA +75 $\mathrm{mmol} / \mathrm{L}$ VPA in TrkB ${ }^{\mathrm{c}-\mathrm{kit}} \mathrm{KO}$ and WT littermate mice. Hematoxylin and eosin stain. B and C: Corresponding quantitative analyses of cell number per section in the GCL (B) and IRL thickness (C). Data are expressed as means \pm SEM. $n=6 .{ }^{*} P<0.05,{ }^{*} P<0.01$, and ${ }^{* * *} P<0.001$. Scale bar $=100 \mu \mathrm{m}$ (upper images) or $50 \mu \mathrm{m}$ (lower images). $\mathrm{GCL}$, ganglion cell layer; INL, inner nuclear layer; IRL, inner retinal layer; K0, knockout; NMDA, N-methyl-Daspartate; $\mathrm{ONL}$, outer nuclear layer; VPA, valproic acid; WT, wild type.

using SD-OCT. The thickness of the ganglion cell complex in $\operatorname{TrkB}^{\mathrm{c}-\mathrm{kit}} \mathrm{KO}$ mice was significantly reduced after NMDA injection; the protective effects of VPA were abolished at day 7 , but a small degree of protection was observed at day 14 (Figure 6). Next, we examined histology of the retina at day 14 in $\mathrm{TrkB}^{\mathrm{c}-\mathrm{kit}} \mathrm{KO}$ mice. VPA only marginally suppressed cell loss in the GCL in TrkB ${ }^{\text {c-kit }}$ KO mice after NMDA injection, and slightly reduced the thinning of the inner retinal layer (Figure 7A). We note that $\mathrm{TrkB}^{\mathrm{c}-\mathrm{kit}} \mathrm{KO}$ mice were more sensitive to NMDA neurotoxicity, compared with WT littermates. Even allowing for this phenotype, the neuroprotective effect of VPA in TrkB $^{\text {c-kit }} \mathrm{KO}$ mice after NMDA injection was significantly smaller than in WT littermates (Figure 7, B and C). These results suggest that neuronal TrkB plays an important role in VPA-mediated neuroprotection against NMDA neurotoxicity in the retina.

\section{VPA Reduces NMDA-Induced 0xidative Stress}

Our findings so far had demonstrated the importance of neuronal BDNF-TrkB signaling. However, the fact that the neuroprotective effects of VPA were not completely abolished in $\mathrm{TrkB}^{\mathrm{c}-\mathrm{kit}} \mathrm{KO}$ mice suggests that there are other pathways for VPA-mediated neuroprotection. One of the major causes of cell death associated with NMDA neurotoxicity is induction of oxidative stress. We therefore investigated if VPA modulates the oxidative stress level after NMDA injection in the retina. We first examined the retinal expression of 4-hydroxy-2-nonenal, which is a common byproduct of lipid peroxidation during oxidative stress and provides a reliable measure of oxidative stress, after NMDA injection with or without VPA. In the NMDAtreated retina, there was a significant increase in expression of 4-hydroxy-2-nonenal in the GCL, compared with the PBS-treated retina, indicative of increased oxidative stress, but 4-hydroxy-2-nonenal expression was significantly suppressed in the presence of VPA (Figure 8, A and B). In addition, the expression of inducible nitric oxide synthase, which is usually induced in an oxidative environment and so is a good indicator of oxidative stress, was significantly increased in the NMDA-treated retina, but this effect was suppressed in the VPA-treated retina (Figure 8C). These results suggest that inhibition of oxidative stress is another mechanism for VPA-mediated neuroprotection against NMDA neurotoxicity in the retina.

\section{Discussion}

In the present study, we have demonstrated that the widely prescribed antiepileptic drug VPA protects RGCs from NMDA-induced neurotoxicity, mainly through stimulation of neuronal BDNF-TrkB signaling. We examined the neuroprotective effects of VPA in terms of both histology and function, and used a noninvasive in vivo imaging technique for monitoring retinal degeneration over time in the same animal. We show that VPA up-regulates BDNF and NGF expression in Müller cells. Using TrkB $^{\text {c-kit }} \mathrm{KO}$ mice, we provide direct evidence that the neuronal TrkB receptor plays an important role in VPA-mediated neuroprotection against
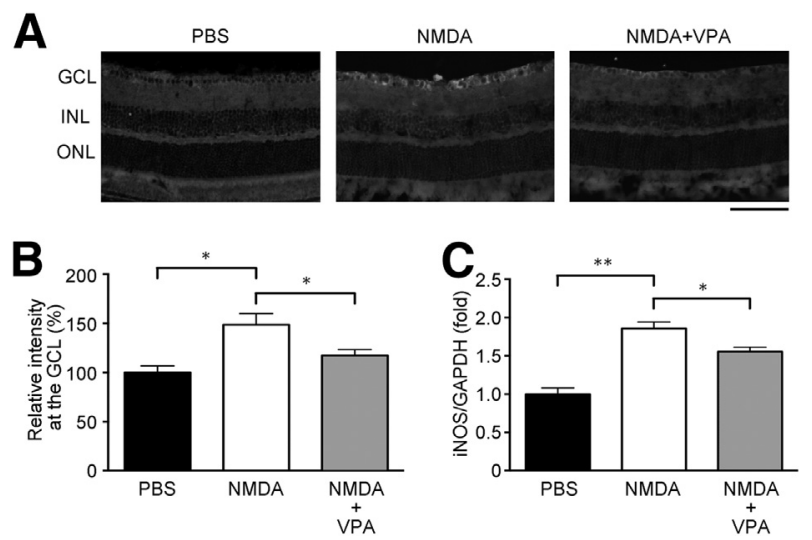

Figure 8 VPA suppresses oxidative stress in the mouse retina after NMDA-induced neurotoxicity. A: Representative images of 4-hydroxy-2nonenal expression in the retina at 12 hours after intravitreal injection of PBS, $1 \mathrm{mmol} / \mathrm{L} \mathrm{NMDA}$, or $1 \mathrm{mmol} / \mathrm{L} \mathrm{NMDA}+75 \mathrm{mmol} / \mathrm{L}$ VPA. Lighter colors indicate higher expression. B: Corresponding quantitative analyses of 4-hydroxy-2-nonenal expression at the GCL. C: Quantitative analyses of relative mRNA expression levels of iNOS in the same retina at 24 hours after intravitreal injection of PBS, $1 \mathrm{mmol} / \mathrm{L} \mathrm{NMDA}$, or $1 \mathrm{mmol} / \mathrm{L} \mathrm{NMDA}+75$ $\mathrm{mmol} / \mathrm{L}$ VPA. GAPDH was used as an internal control and results are normalized to controls (GAPDH mRNA expression). Data are expressed as means \pm SEM. $n=4 .{ }^{*} P<0.05,{ }^{*} P<0.01$. Scale bar $=100 \mu \mathrm{m} . \mathrm{GCL}$, ganglion cell layer; INL, inner nuclear layer; iNOS, inducible nitric oxide synthase; NMDA, N-methyl-D-aspartate; ONL, outer nuclear layer; PBS, phosphate-buffered saline; VPA, valproic acid. 
NMDA neurotoxicity in the retina. We also show an alternative neuroprotective mechanism in which VPA suppresses oxidative stress after induction of NMDA neurotoxicity in the retina.

VPA is an effective histone deacetylase inhibitor, ${ }^{6,7}$ and treatment with VPA increases levels of neurotrophic factors in mouse and rat brain. ${ }^{40-42}$ In the retina, VPA upregulated the expression of BDNF and TrkB after optic nerve injury, and there was an increased acetylation level of histone $\mathrm{H} 3$ accompanied by increased transcriptional activity of the BDNF promoter in the retina. ${ }^{43}$ These results suggest that the VPA-mediated up-regulation of BDNF in Müller cells observed in the present study is likely to be due to elevated BDNF promotor activity through direct inhibition of histone deacetylase. Neuroprotection by stimulation of BDNF-TrkB signaling has been well established. Interestingly, $\operatorname{TrkB}^{\mathrm{c}-\mathrm{kit}} \mathrm{KO}$ mice demonstrated increased susceptibility to NMDA-induced neurotoxicity (Figures 6 and 7), ${ }^{33}$ indicating the importance of the intrinsic BDNF-TrkB signaling pathway in neuroprotection in the retina. Recently, several selective TrkB agonists have been proposed as therapeutic agents for neurodegenerative diseases. ${ }^{44,45}$ Based on the evidence that neurotrophic factors protect the retina from various pathological conditions, ${ }^{46}$ development of such compounds that mimic some of the beneficial effects of neurotrophic factors is of clinical interest. Interestingly, a novel phosphine-borane complex was reported to promote $\mathrm{RGC}$ protection through induction of BDNF, ${ }^{47}$ which supports our present findings and also supports the idea that up-regulation of BDNF and increased signaling through TrkB by VPA may be effective for treatment of glaucoma.

Müller cells are the principal glial cells in the retina, and recent studies show that their roles extend beyond supporting retinal structure and homeostasis. ${ }^{48,49}$ For example, prolonged gene delivery of BDNF in Müller cells enhanced neuronal survival after optic nerve injury. ${ }^{50} \mathrm{We}$ have previously reported important roles of neurotrophic factors in Müller cells in protection of photoreceptors and RGCs. ${ }^{23,33,36}$ Our present findings suggest a mechanism in which administration of VPA up-regulates BDNF in Müller cells, which in turn stimulates neuronal BDNF-TrkB signaling and thus leads to RGC protection against NMDA-induced neurotoxicity. In addition, our present results demonstrated that VPA increases NGF production in Müller cells. The delay of 48 hours in up-regulation of NGF suggests that its neuroprotective effects on acute RGC death may be limited, but that it may be effective in protecting RGCs from later death. In a recent study, NGF eye drops reduced RGC loss in patients with advanced glaucoma, and longlasting improvements were observed in visual field, optic nerve function, contrast sensitivity, and visual acuity. ${ }^{51}$ Although further studies are required before topical NGF can be considered for clinical application in glaucoma, VPA-induced NGF may be beneficial for RGC protection in human eyes. We have previously demonstrated that brimonidine eye drops stimulate production of NGF, BDNF, and basic fibroblast growth factor in Müller cells and ameliorate retinal degeneration in a murine model of normal tension glaucoma. ${ }^{23}$ Thus, in addition to lowering intraocular pressure, combination treatment with VPA and brimonidine may prevent glaucomatous retinal degeneration by stimulating multiple pathways, including glia-neuron interactions.

Oxidative stress is an important risk factor in human glaucoma, ${ }^{52}$ and studies with DBA/2J mice, an animal model that recapitulates the slow and progressive nature of human glaucoma, demonstrated that the antioxidant $\alpha$-lipoic acid protects RGCs in the glaucomatous retina. ${ }^{53}$ VPA has been reported to enhance activities of several enzymes with antioxidant properties in the ischemic retina. ${ }^{54}$ Consistent with our present findings (Figure 8), antioxidant properties of VPA have been demonstrated in brain and retina in a stroke model ${ }^{55,56}$ and also in spinal cord injury. ${ }^{57}$ These studies support our conclusion that suppression of oxidative stress is one of the neuroprotective mechanisms for VPA in the NMDA excitotoxicity model.

We note some limitations to the present study. First, the neuroprotective action of VPA involves multiple mechanisms. ${ }^{58}$ Although our findings in $\mathrm{TrkB}^{\mathrm{c}-\mathrm{kit}} \mathrm{KO}$ mice suggest that the BDNF-TrkB signaling pathway plays a major role in VPA-mediated neuroprotective effects on NMDA-induced neurotoxicity in the retina, and although we also demonstrated increased glial NGF expression and reduced oxidative stress, we cannot eliminate the possibility that other protective mechanisms are also involved. One possible mechanism may be a direct effect of VPA on neuronal excitability. VPA inhibits the activity of voltagegated cation channels, ${ }^{59,60}$ suggesting that NMDA neurotoxicity in the present study may also be suppressed through this mechanism. Thus, our present results do not fully explain the neuroprotective mechanisms associated with VPA. Second, the NMDA excitotoxicity model mimics some key features observed in human glaucoma, including loss of RGCs and increased oxidative stress, but the underlying molecular mechanisms are not quite the same as for pathogenesis of human glaucoma. Previous studies have indicated that VPA protects RGCs from optic nerve injury-induced cell death. ${ }^{43,61}$ The optic nerve injury model is an animal model of glaucoma that allows investigation of mechanisms underlying death of retinal neurons, ${ }^{34,62}$ but this model is not sufficient to represent fully the pathogenesis of glaucoma. It would therefore be worthwhile to investigate if VPA can ameliorate glaucomatous retinal degeneration in other animal models of glaucoma.

In summary, we have shown that VPA suppresses RGC death from NMDA-induced neurotoxicity and we provide direct evidence for the role of neuronal TrkB in VPAmediated neuroprotection. Our findings emphasize the importance of glia-neuron crosstalk in neuroprotection and raise an interesting possibility that VPA may be effective for treatment of glaucoma. 


\section{Acknowledgments}

We thank Mayumi Kunitomo, Yuriko Azuchi, and Keiko Okabe for technical assistance.

\section{References}

1. Pinder RM, Brogden RN, Speight TM, Avery GS: Sodium valproate: a review of its pharmacological properties and therapeutic efficacy in epilepsy. Drugs 1977, 13:81-123

2. Chiu CT, Wang Z, Hunsberger JG, Chuang DM: Therapeutic potential of mood stabilizers lithium and valproic acid: beyond bipolar disorder. Pharmacol Rev 2013, 65:105-142

3. Hoffmann J, Akerman S, Goadsby PJ: Efficacy and mechanism of anticonvulsant drugs in migraine. Expert Rev Clin Pharmacol 2014, $7: 191-201$

4. Waszkielewicz AM, Gunia A, Sloczyńska K, Marona H: Evaluation of anticonvulsants for possible use in neuropathic pain. Curr Med Chem 2011, 18:4344-4358

5. Gurvich N, Klein PS: Lithium and valproic acid: parallels and contrasts in diverse signaling contexts. Pharmacol Ther 2002, 96: 45-66

6. Göttlicher M, Minucci S, Zhu P, Krämer OH, Schimpf A, Giavara S, Sleeman JP, Lo Coco F, Nervi C, Pelicci PG, Heinzel T: Valproic acid defines a novel class of HDAC inhibitors inducing differentiation of transformed cells. EMBO J 2001, 20:6969-6978

7. Phiel CJ, Zhang F, Huang EY, Guenther MG, Lazar MA, Klein PS: Histone deacetylase is a direct target of valproic acid, a potent anticonvulsant, mood stabilizer, and teratogen. J Biol Chem 2001, 276: 36734-36741

8. Chen S, Wu H, Klebe D, Hong Y, Zhang J: Valproic Acid: a new candidate of therapeutic application for the acute central nervous system injuries. Neurochem Res 2014, 39:1621-1633

9. Monti B, Gatta V, Piretti F, Raffaelli SS, Virgili M, Contestabile A: Valproic acid is neuroprotective in the rotenone rat model of Parkinson's disease: involvement of alpha-synuclein. Neurotox Res 2010, 17:130-141

10. Qing H, He G, Ly PT, Fox CJ, Staufenbiel M, Cai F, Zhang Z, Wei S, Sun X, Chen CH, Zhou W, Wang K, Song W: Valproic acid inhibits Abeta production, neuritic plaque formation, and behavioral deficits in Alzheimer's disease mouse models. J Exp Med 2008, 205: $2781-2789$

11. Zhang XZ, Li XJ, Zhang HY: Valproic acid as a promising agent to combat Alzheimer's disease. Brain Res Bull 2010, 81:3-6

12. Long Z, Zheng M, Zhao L, Xie P, Song C, Chu Y, Song W, He G: Valproic acid attenuates neuronal loss in the brain of APP/PS1 double transgenic Alzheimer's disease mice model. Curr Alzheimer Res 2013, 10:261-269

13. Quigley HA, Broman AT: The number of people with glaucoma worldwide in 2010 and 2020. Br J Ophthalmol 2006, 90:262-267

14. John SW, Smith RS, Savinova OV, Hawes NL, Chang B, Turnbull D, Davisson M, Roderick TH, Heckenlively JR: Essential iris atrophy, pigment dispersion, and glaucoma in $\mathrm{DBA} / 2 \mathrm{~J}$ mice [Erratum appeared in Invest Ophthalmol Vis Sci 1998, 39:1641]. Invest Ophthalmol Vis Sci 1998, 39:951-962

15. WoldeMussie E, Ruiz G, Wijono M, Wheeler LA: Neuroprotection of retinal ganglion cells by brimonidine in rats with laser-induced chronic ocular hypertension. Invest Ophthalmol Vis Sci 2001, 42: 2849-2855

16. Garcia-Valenzuela E, Shareef S, Walsh J, Sharma SC: Programmed cell death of retinal ganglion cells during experimental glaucoma. Exp Eye Res 1995, 61:33-44

17. Morrison JC, Moore CG, Deppmeier LM, Gold BG, Meshul CK, Johnson EC: A rat model of chronic pressure-induced optic nerve damage. Exp Eye Res 1997, 64:85-96
18. Klein BE, Klein R, Sponsel WE, Franke T, Cantor LB, Martone J, Menage MJ: Prevalence of glaucoma. The Beaver Dam Eye Study. Ophthalmology 1992, 99:1499-1504

19. Iwase A, Suzuki Y, Araie M, Yamamoto T, Abe H, Shirato S, Kuwayama Y, Mishima HK, Shimizu H, Tomita G, Inoue Y, Kitazawa Y; Tajimi Study Group, Japan Glaucoma Society: The prevalence of primary open-angle glaucoma in Japanese: the Tajimi Study. Ophthalmology 2004, 111:1641-1648

20. Harada T, Harada C, Nakamura K, Quah HM, Okumura A, Namekata K, Saeki T, Aihara M, Yoshida H, Mitani A, Tanaka K: The potential role of glutamate transporters in the pathogenesis of normal tension glaucoma. J Clin Invest 2007, 117:1763-1770

21. Harada C, Namekata K, Guo X, Yoshida H, Mitamura Y, Matsumoto Y, Tanaka K, Ichijo H, Harada T: ASK1 deficiency attenuates neural cell death in GLAST-deficient mice, a model of normal tension glaucoma. Cell Death Differ 2010, 17:1751-1759

22. Semba K, Namekata K, Guo X, Harada C, Harada T, Mitamura Y: Renin-angiotensin system regulates neurodegeneration in a mouse model of normal tension glaucoma. Cell Death Dis 2014, 5:e1333

23. Semba K, Namekata K, Kimura A, Harada C, Mitamura Y, Harada T: Brimonidine prevents neurodegeneration in a mouse model of normal tension glaucoma. Cell Death Dis 2014, 5:e1341

24. Li Y, Schlamp CL, Nickells RW: Experimental induction of retinal ganglion cell death in adult mice. Invest Ophthalmol Vis Sci 1999, 40:1004-1008

25. Namekata K, Kimura A, Kawamura K, Guo X, Harada C, Tanaka K, Harada T: Dock3 attenuates neural cell death due to NMDA neurotoxicity and oxidative stress in a mouse model of normal tension glaucoma. Cell Death Differ 2013, 20:1250-1256

26. Cordeiro MF, Guo L, Luong V, Harding G, Wang W, Jones HE, Moss SE, Sillito AM, Fitzke FW: Real-time imaging of single nerve cell apoptosis in retinal neurodegeneration. Proc Natl Acad Sci USA 2004, 101:13352-13356

27. Schuettauf F, Quinto K, Naskar R, Zurakowski D: Effects of antiglaucoma medications on ganglion cell survival: the DBA/2J mouse model. Vision Res 2002, 42:2333-2337

28. Seki M, Lipton SA: Targeting excitotoxic/free radical signaling pathways for therapeutic intervention in glaucoma. Prog Brain Res 2008, 173:495-510

29. Osborne NN: Recent clinical findings with memantine should not mean that the idea of neuroprotection in glaucoma is abandoned. Acta Ophthalmol 2009, 87:450-454

30. Cheng L, Sapieha P, Kittlerova P, Hauswirth WW, Di Polo A: TrkB gene transfer protects retinal ganglion cells from axotomy-induced death in vivo. J Neurosci 2002, 22:3977-3986

31. Parrilla-Reverter G, Agudo M, Sobrado-Calvo P, Salinas-Navarro M, Villegas-Pérez MP, Vidal-Sanz M: Effects of different neurotrophic factors on the survival of retinal ganglion cells after a complete intraorbital nerve crush injury: a quantitative in vivo study. Exp Eye Res 2009, 89:32-41

32. Martin KR, Quigley HA, Zack DJ, Levkovitch-Verbin H, Kielczewski J, Valenta D, Baumrind L, Pease ME, Klein RL, Hauswirth WW: Gene therapy with brain-derived neurotrophic factor as a protection: retinal ganglion cells in a rat glaucoma model. Invest Ophthalmol Vis Sci 2003, 44:4357-4365

33. Harada C, Guo X, Namekata K, Kimura A, Nakamura K, Tanaka K, Parada LF, Harada T: Glia- and neuron-specific functions of TrkB signalling during retinal degeneration and regeneration. Nat Commun 2011, 2:189

34. Katome T, Namekata K, Guo X, Semba K, Kittaka D, Kawamura K, Kimura A, Harada C, Ichijo H, Mitamura Y, Harada T: Inhibition of ASK1-p38 pathway prevents neural cell death following optic nerve injury. Cell Death Differ 2013, 20:270-280

35. Harada C, Nakamura K, Namekata K, Okumura A, Mitamura Y, lizuka Y, Kashiwagi K, Yoshida K, Ohno S, Matsuzawa A, Tanaka K, Ichijo H, Harada T: Role of apoptosis signal-regulating 
kinase 1 in stress-induced neural cell apoptosis in vivo. Am J Pathol 2006, 168:261-269

36. Harada T, Harada C, Kohsaka S, Wada E, Yoshida K, Ohno S, Mamada H, Tanaka K, Parada LF, Wada K: Microglia-Müller glia cell interactions control neurotrophic factor production during lightinduced retinal degeneration. J Neurosci 2002, 22:9228-9236

37. Guo X, Nakamura K, Kohyama K, Harada C, Behanna HA, Watterson DM, Matsumoto Y, Harada T: Inhibition of glial cell activation ameliorates the severity of experimental autoimmune encephalomyelitis. Neurosci Res 2007, 59:457-466

38. Sutter EE, Bearse MA Jr: The optic nerve head component of the human ERG. Vision Res 1999, 39:419-436

39. Bearse MA Jr, Sutter EE, Sim D, Stamper R: Glaucomatous dysfunction revealed in higher order components of the electroretinogram. Vision Science and Its Applications. OSA Technical Digest Series. Washington, DC, Optical Society of America, 1996, pp 105-107

40. Chen PS, Peng GS, Li G, Yang S, Wu X, Wang CC, Wilson B, Lu RB, Gean PW, Chuang DM, Hong JS: Valproate protects dopaminergic neurons in midbrain neuron/glia cultures by stimulating the release of neurotrophic factors from astrocytes. Mol Psychiatry 2006, $11: 1116-1125$

41. Yasuda S, Liang MH, Marinova Z, Yahyavi A, Chuang DM: The mood stabilizers lithium and valproate selectively activate the promoter IV of brain-derived neurotrophic factor in neurons. Mol Psychiatry 2009, 14:51-59

42. Noh H, Seo H: Age-dependent effects of valproic acid in Alzheimer's disease $(\mathrm{AD})$ mice are associated with nerve growth factor (NGF) regulation. Neuroscience 2014, 266:255-265

43. Zhang ZZ, Gong YY, Shi YH, Zhang W, Qin XH, Wu XW: Valproate promotes survival of retinal ganglion cells in a rat model of optic nerve crush. Neuroscience 2012, 224:282-293

44. Massa SM, Yang T, Xie Y, Shi J, Bilgen M, Joyce JN, Nehama D, Rajadas J, Longo FM: Small molecule BDNF mimetics activate TrkB signaling and prevent neuronal degeneration in rodents. J Clin Invest 2010, 120:1774-1785

45. Jang SW, Liu X, Yepes M, Shepherd KR, Miller GW, Liu Y, Wilson WD, Xiao G, Blanchi B, Sun YE, Ye K: A selective TrkB agonist with potent neurotrophic activities by 7,8-dihydroxyflavone. Proc Natl Acad Sci USA 2010, 107:2687-2692

46. Harada T, Harada C, Parada LF: Molecular regulation of visual system development: more than meets the eye. Genes Dev 2007, 21: 367-378

47. Almasieh M, Lieven CJ, Levin LA, Di Polo A: A cell-permeable phosphine-borane complex delays retinal ganglion cell death after axonal injury through activation of the pro-survival extracellular signal-regulated kinases 1/2 pathway. J Neurochem 2011, 118: $1075-1086$

48. Bringmann A, Iandiev I, Pannicke T, Wurm A, Hollborn M, Wiedemann P, Osborne NN, Reichenbach A: Cellular signaling and factors involved in Müller cell gliosis: neuroprotective and detrimental effects. Prog Retin Eye Res 2009, 28:423-451
49. Goldman D: Müller glial cell reprogramming and retina regeneration. Nat Rev Neurosci 2014, 15:431-442

50. Di Polo A, Aigner LJ, Dunn RJ, Bray GM, Aguayo AJ: Prolonged delivery of brain-derived neurotrophic factor by adenovirus-infected Müller cells temporarily rescues injured retinal ganglion cells. Proc Natl Acad Sci USA 1998, 95:3978-3983

51. Lambiase A, Aloe L, Centofanti M, Parisi V, Mantelli F, Colafrancesco V, Manni GL, Bucci MG, Bonini S, LeviMontalcini R: Experimental and clinical evidence of neuroprotection by nerve growth factor eye drops: implications for glaucoma. Proc Natl Acad Sci USA 2009, 106:13469-13474

52. Goyal A, Srivastava A, Sihota R, Kaur J: Evaluation of oxidative stress markers in aqueous humor of primary open angle glaucoma and primary angle closure glaucoma patients. Curr Eye Res 2014, 39: 823-829

53. Inman DM, Lambert WS, Calkins DJ, Horner PJ: alpha-Lipoic acid antioxidant treatment limits glaucoma-related retinal ganglion cell death and dysfunction. PLoS One 2013, 8:e65389

54. Zhang Z, Qin X, Zhao X, Tong N, Gong Y, Zhang W, Wu X: Valproic acid regulates antioxidant enzymes and prevents ischemia/ reperfusion injury in the rat retina. Curr Eye Res 2012, 37:429-437

55. Suda S, Katsura K, Kanamaru T, Saito M, Katayama Y: Valproic acid attenuates ischemia-reperfusion injury in the rat brain through inhibition of oxidative stress and inflammation. Eur J Pharmacol 2013, 707:26-31

56. Zhang Z, Tong N, Gong Y, Qiu Q, Yin L, Lv X, Wu X: Valproate protects the retina from endoplasmic reticulum stress-induced apoptosis after ischemia-reperfusion injury. Neurosci Lett 2011, 504:88-92

57. Lee JY, Maeng S, Kang SR, Choi HY, Oh TH, Ju BG, Yune TY: Valproic acid protects motor neuron death by inhibiting oxidative stress and endoplasmic reticulum stress-mediated cytochrome C release after spinal cord injury. J Neurotrauma 2014, 31:582-594

58. Monti B, Polazzi E, Contestabile A: Biochemical, molecular and epigenetic mechanisms of valproic acid neuroprotection. Curr Mol Pharmacol 2009, 2:95-109

59. VanDongen AM, VanErp MG, Voskuyl RA: Valproate reduces excitability by blockage of sodium and potassium conductance. Epilepsia 1986, 27:177-182

60. Tian LM, Alkadhi KA: Valproic acid inhibits the depolarizing rectification in neurons of rat amygdala. Neuropharmacology 1994, $33: 1131-1138$

61. Biermann J, Grieshaber P, Goebel U, Martin G, Thanos S, Di Giovanni S, Lagrèze WA: Valproic acid-mediated neuroprotection and regeneration in injured retinal ganglion cells. Invest Ophthalmol Vis Sci 2010, 51:526-534

62. Semba K, Namekata K, Kimura A, Harada C, Katome T, Yoshida H, Mitamura Y, Harada T: Dock3 overexpression and p38 MAPK inhibition synergistically stimulate neuroprotection and axon regeneration after optic nerve injury. Neurosci Lett 2014, 581:89-93 